\title{
Conocimientos, creencias y manejo de la fiebre entre los padres consultantes en un Hospital Pediátrico de Tercer nivel en Pasto, Colombia, 2017
}

\author{
Knowledge, beliefs and management of fever among parents \\ consulting at a Third Level Pediatric Hospital in Pasto, \\ Colombia, 2017
}

\author{
Diana Vanessa Suarez Ayala' ${ }^{1}$ Nancy Cabrera', Alejandra Delgado'1 Sandra López ${ }^{1}$
}

\begin{abstract}
RESUMEN
Introducción: En Colombia existen muchos mitos, y se observa diferentes abordajes de la fiebre. Sin embargo, no hay datos locales que muestren esta situación. El Objetivo de este estudio es Identificar los conocimientos, creencias, y manejos de la fiebre entre los padres consultantes. Materiales y Métodos: Estudio descriptivo, observacional, transversal, se aplicó un cuestionario a una muestra tomada conveniencia de 200 padres que consultaron al servicio de urgencia del hospital. Resultados: Se realizaron 200 encuestas. El 59\% de los padres se encontraban en secundaria. El $84,5 \%$ de la población pertenecía a los estratos 1 y 2 y vivía en zona urbana. El 52\% utilizaban termómetros o tiras plásticas para medir la temperatura. El $81,5 \%$ administró antibiótico solo cuando el médico lo prescribe. Las variables sociodemográficas de los padres, que tuvieron una relación estadísticamente significativa se relacionaron en el conocimiento, manejo y creencias de la fiebre fueron: edad de los padres, nivel educativo, estrato socieconomico y número de hijos. Conclusiones: Se encontraron falencias en el conocimiento de los padres en conceptos de fiebre y toma de temperatura. Los padres se basan en consultas previas al médico para la administración de medicamentos. En el manejo de la fiebre, la mayoría de padres utilizan medios físicos para disminuirla concomitante con el uso de antipiréticos; con respecto a las creencias, en nuestra región (Pasto - Nariño) siguen siendo utilizadas las terapias tradicionales para el manejo de fiebre.
\end{abstract}

Palabras Clave: Fiebre, creencias, antipiréticos, manejo, padres.

\begin{abstract}
Introduction: In Colombia there are many myths, and different approaches to fever are observed. However, there is no local data describing this situation. The objective of this study is to identify the knowledge, beliefs, and management of fever among parents requesting consultation. Materials and Methods: This was a descriptive, observational and cross-sectional study. A questionnaire was applied to a convenience sample of 200 parents who consulted at the emergency department of the hospital. Results: 200 surveys were carried out. $59 \%$ of parents were in high school. $84.5 \%$ of the population belonged to stratas 1 and 2 and lived in an urban area. 52\% used thermometers or plastic strips to measure the temperature. $81.5 \%$ administered antibiotics only when the doctor prescribed it. The sociodemographic variables of the parents, which were significantly statistically e related to the knowledge, management and beliefs of the fever were: age of the parents, educational level, socioeconomic status and number of children. Conclusions: We found knowledge gaps in parents regarding fever and temperature measurement. Parents administer medications after consulting with a physician. In managing fever, most parents used physical means to reduce fever along with antipyretics. Regarding beliefs, in our region (Pasto - Nariño) traditional therapies for the management of fever are still used.
\end{abstract}

Keywords: Fever, beliefs, antipyretics, management, parents.

\footnotetext{
${ }^{1}$ Fundación Hospital Infantil Los Ángeles. San Juan de Pasto, Colombia. Correspondencia: Diana Vanessa Suarez Ayala Correo: divane30@gmail.com Conflicto de interés: Los autores declaran no poseer conflicto de interés Recibido: 03/11/2018; Aceptado: 12/12/2018 DOI: https://doi.org/10.31698/ped.45032018007
} 


\section{INTRODUCCIÓN}

En 1980 el Dr. Schmitt fue el primero en acuñar el término "fiebrefobia" ó "fobia a la fiebre" para describir los temores infundados de los padres sobre la fiebre: este término ésta asociado con numerosos conceptos erróneos acerca de su papel en las diferentes enfermedades esta fobia se ha generalizado a tal punto que incluso, se encuentra entre los profesionales de la salud, en todo el mundo ${ }^{(1,2)}$.

Aunque la fiebre se consideró una respuesta protectora por muchos años, el uso de fármacos antipiréticos ha llevado a la creencia común de que la fiebre es desadaptativa y perjudicial. Los estudios han mostrado que los padres pueden tener varios conceptos errados y creencias acerca de la fiebre, su papel en las diferentes enfermedades según la etiología que las produzca, y así mismo, su manejo. Existen variaciones raciales y étnicas en estas

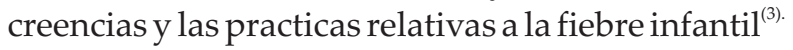
La fiebre en sí misma no es una enfermedad, por el contrario es un método por el cual el cuerpo combate las infecciones, causado por virus y bacterias o puede tener causas no infecciosas ${ }^{(4)}$. La fiebre aumenta la proliferación de la producción de neutrófilos y linfocitos $\mathrm{T}$, ayudando a la reacción de la fase aguda del cuerpo. Se han realizado estudios en diferentes poblaciones que muestran diferentes asociaciones entre variables como por ejemplo el nivel socioeconómico y educativo de los padres, con el conocimiento general de la fiebre ${ }^{(5,6)}$.

En Colombia existen muchos mitos, y se observan diferentes abordajes de la fiebre. Sin embargo, no hay datos locales que muestren esta situación, por lo que al actual estudio tiene como objetivo identificar los conocimientos, creencias, y manejos de la fiebre entre los padres consultantes en un Hospital Pediátrico de tercer nivel de complejidad en Pasto, Colombia.

\section{MATERIALES Y MÉTODOS}

Se realizó un estudio descriptivo, observacional, transversal. Fueron incluidos los padres de niños con diagnóstico de fiebre que consultaron al servicio de Urgencias Pediátricas del Hospital, entre julio 2017 a diciembre de 2017.
Se aplicó una encuesta de 18 preguntas de opciones cerradas y abiertas, tiendo en cuenta variables sociodemográficas tales como: Edad, número de hijos, estado civil, ocupación, nivel de educación, estrato socioeconómico y lugar de residencia. Este instrumento de recolección de información fue validado, tomada del estudio publicado en el artículo: "Parents' Knowledge, Attitudes and Beliefsof ChildhoodFever Management in Jordan: a Cross SectionalStudy", autorizada por sus autores ${ }^{(3)}$. Donde se midió conocimientos, creencias y manejo de la fiebre, la cual fue aplicada a un total de 200 padres. Se validó por medio de la realización de una prueba piloto registrando y analizando los primeros 20 casos de dicho evento. Con el fin de evaluar la capacidad, disponibilidad de información y validez, estos no se incluyeron dentro del estudio.

Para la selección de la muestra se hizo uso del muestreo aleatorio donde todos y cada uno de los elementos de la población tengan la misma probabilidad de ser seleccionados. Considerando que la población del estudio está determinada cuantitativamente. Se controlaron sesgos de selección estableciendo criterios de inclusión: padres que consultaron al servicio de urgencias por que el menor a su cuidado presentaba fiebre. Exclusión: a padres que no hablen español y que tuvieran patología mental que pueda afectar las respuestas de la encuesta. El estudio fue avalado por el Comité de ética en la Investigación del Hospital donde se desarrolló.

\section{Análisis estadístico}

Para tabulación y análisis de la información se utilizó el software SPSS ${ }^{\oplus}$, versión 21 (IBM Corp. Released 2012. IBM SPSS Statistics for Windows, Version 21.0. Armonk, NY: IBM Corp.). Se realizó un análisis descriptivo de la población, según la naturaleza de las variables de estudio, Se realizó la descripción de las características sociodemográficas de la población, teniendo en cuenta la naturaleza de las variables: para las variables cualitativas se utilizó frecuencias absolutas y relativas. Para variables cuantitativas se empleó el promedio, desviación estándar, mediana y rango intercuartil y se probó el 
supuesto de normalidad con la prueba de Kolmogorov-Smirnov. Posteriormente se llevó a cabo un análisis bivariado para estimar la relación entre variables cualitativas, donde se aplicó Chi cuadrado de independencia.

\section{RESULTADOS}

Se realizaron en total 200 encuestas a padres que consultaron al hospital. El 42,5 \% (85) de los padres se encontraban en edades entre 25 y 33 años, la gran mayoría 51,5\% (103) tenía un hijo. En cuanto al estado civil el $43 \%$ (86) se encontraban en unión libre. Llama la atención que el 64,5\% (129) de los padres en el momento de aplicar la encuesta se encontraba desempleados. Para el nivel educativo el $59 \%$ (118) se encontraban en secundaria. El 84,5\% (169) de nuestra población pertenecía a los estratos 1 y 2 y vivía en zona urbana. Se aclara que, en Colombia, según el DANE (Departamento Administrativo Nacional de Estadísticas) se clasifica el estrato socioeconómico entre 1 y 6 , siendo el estrato 1 el más bajo (Tabla 1).

Tabla 1. Características sociodemográficas en el servicio de urgencias.

\begin{tabular}{|c|c|c|c|}
\hline Características & Característica & Frecuencia & $(\%)$ \\
\hline \multirow[t]{4}{*}{ Edad (del padre o madre) } & $16-24$ & 68 & 34 \\
\hline & $25-33$ & 85 & 42,5 \\
\hline & $34-41$ & 32 & 16 \\
\hline & Mayor de 42 & 15 & 7,5 \\
\hline \multirow[t]{3}{*}{ Número de hijos } & 1 hijo & 103 & 51,5 \\
\hline & 2-3 hijos & 83 & 41,5 \\
\hline & Más de 4 hijos & 14 & 7 \\
\hline \multirow[t]{4}{*}{ Estado civil } & Casado & 47 & 23,5 \\
\hline & Unión libre & 86 & 43 \\
\hline & Soltero & 65 & 32,5 \\
\hline & Viudo & 2 & 1 \\
\hline \multirow[t]{2}{*}{ Ocupación } & Empleado & 71 & 35,5 \\
\hline & Desempleado & 129 & 64,5 \\
\hline \multirow[t]{3}{*}{ Nivel de educación } & Primaria & 43 & 21,5 \\
\hline & Secundaria & 118 & 59 \\
\hline & Universitario & 39 & 19,5 \\
\hline \multirow[t]{2}{*}{ Estrato socioeconómico } & Estrato 1-2 & 169 & 84,5 \\
\hline & Estrato 3-4 & 31 & 15,5 \\
\hline \multirow[t]{2}{*}{ Lugar de residencia } & Urbano & 169 & 84,5 \\
\hline & Rural & 31 & 15,5 \\
\hline
\end{tabular}

Para el análisis de los resultados se dividieron las preguntas de la encuesta en tres categorías: Conocimientos, manejo y creencias.
En cuanto a los conocimientos (Tabla 2), El 52\% (104) de los padres utilizan termómetros o tiras plásticas para medir la temperatura de sus hijos en casa, sin embargo, un porcentaje importante $46 \%$ (92), utilizan la mano como medida subjetiva de presencia de fiebre. En cuanto al sitio anatomía la axila, con el $81,5 \%$ (163) es el lugar más frecuente para toma de temperatura. El 54\% (108) de los padres conocen que la definición de fiebre es una temperatura mayor a $38^{\circ} \mathrm{C}$ y el $75,5 \%$ (151) refiere que consulta a su médico cuando presenta esta temperatura. El acetaminofén es el medicamento más utilizado con un 95\% (190) para el manejo de la fiebre. El 70,5\% (141) de los padres deciden que medicamento y que dosis usar basados en una consulta previa con pediatra o médico general. El 81,5\% (163) de los padres administra antibiótico solo cuando el médico lo prescribe.

Tabla 2. Conocimientos de los padres sobre la fiebre en el servicio de urgencias.

\begin{tabular}{|c|c|c|c|}
\hline Pregunta & Categoría & Frecuencia & $(\%)$ \\
\hline \multirow{4}{*}{$\begin{array}{l}\text { En su casa, se mide la } \\
\text { temperatura de su hijo } \\
\text { enfermo mediante el uso de: }\end{array}$} & Termómetro o tira plástica & 104 & 52 \\
\hline & Su mano & 92 & 46 \\
\hline & No mide la temperatura de su hijo & 2 & 1 \\
\hline & No sé & 2 & 1 \\
\hline \multirow{4}{*}{$\begin{array}{l}\text { ¿Cuándo usted toma una } \\
\text { temperatura de un niño } \\
\text { menor de seis años, cual es } \\
\text { el mejor lugar? }\end{array}$} & Axila & 163 & 81,5 \\
\hline & Boca & 18 & 9 \\
\hline & Recto & 6 & 3 \\
\hline & No sé & 13 & 6,5 \\
\hline \multirow{3}{*}{$\begin{array}{l}\text { ¿Por encima de que } \\
\text { temperatura considera usted } \\
\text { que su niño tenga fiebre? }\end{array}$} & Mayor de 38 & 108 & 54 \\
\hline & $36-37.9$ & 64 & 32 \\
\hline & No sé & 28 & 14 \\
\hline \multirow{3}{*}{$\begin{array}{l}\text { Si su hijo tiene fiebre alta. } \\
\text { ¿Hasta cuanto espera antes } \\
\text { de llamar o ir al médico? }\end{array}$} & $>38$ & 151 & 75,5 \\
\hline & $<38$ & 14 & 7 \\
\hline & No sabe/no aplica & 35 & 17,5 \\
\hline \multirow{4}{*}{$\begin{array}{l}\text { ¿Qué medicamentos } \\
\text { usted da a su hijo? }\end{array}$} & Acetaminofén & 190 & 95 \\
\hline & Ibuprofeno & 7 & 3,5 \\
\hline & Antibiótico & 2 & 1 \\
\hline & Aspirina & 1 & 0,5 \\
\hline \multirow{5}{*}{$\begin{array}{l}\text { ¿Cómo decide el } \\
\text { medicamento correcto para } \\
\text { bajar la fiebre de su hijo? }\end{array}$} & Recomendación previa del pediatra & a 141 & 70,5 \\
\hline & Decido por mí mismo & 19 & 9,5 \\
\hline & Llamo a mi pediatra & 5 & 2,5 \\
\hline & Consulto al farmacéutico & 16 & 8 \\
\hline & Consulto a otras personas & 19 & 9,5 \\
\hline \multirow{5}{*}{$\begin{array}{l}\text { ¿Cómo se calcula la dosis } \\
\text { correcta de medicamentos } \\
\text { para bajar la fiebre a su hijo? }\end{array}$} & Recomendación previa del pediatra & a 141 & 70,5 \\
\hline & Decido por mí mismo & 10 & 5 \\
\hline & Llamo a mi pediatra & 6 & 3 \\
\hline & Consulta al farmacéutico & 22 & 11 \\
\hline & Consulta a otras personas & 21 & 10,5 \\
\hline \multirow{3}{*}{$\begin{array}{l}\text { Usted le administra } \\
\text { antibiótico a su hijo } \\
\text { cuando: }\end{array}$} & Cuando el médico lo indica & 163 & 81,5 \\
\hline & Tiene fiebre & 18 & 9 \\
\hline & Usted sospecha una infección & 19 & 9,5 \\
\hline
\end{tabular}

Para el manejo de la fiebre (Tabla 3), cuando se preguntó: ¿Que otros recursos diferentes a la 
administración de medicamentos usa para bajarles la fiebre a sus hijos?, refirieron que el $81 \%$ (163) utilizan medios físicos (44\% paños de agua tibia y el $37 \%$ baños con agua fría o tibia). El 53\% (106) de los padres usan la cuchara o jeringa especifica que viene con el medicamento para dosificar la cantidad de antipirético. A la pregunta si los padres dan antibiótico a sus hijos enfermos sin consultar al médico, el 87\% (175) respondieron NO, sin embargo, aunque el porcentaje es menor solo el $12,5 \%$ (25) respondieron que si daban antibiótico sin consultar al médico; además se encontró estadísticas similares a las preguntas si los padres daban antibiótico prescrito por el farmaceuta quienes respondieron NO en un 84 \% (169). El 91\% (182) respondieron igualmente que no insiste en la prescripción al médico de antibióticos en caso de que el menor no lo necesita.

Tabla 3. Manejo de la fiebre por parte de los padres en el servicio de urgencias.

\begin{tabular}{|c|c|c|c|}
\hline Pregunta & Categoría & Frecuencia & $(\%)$ \\
\hline \multirow{6}{*}{$\begin{array}{l}\text { ¿Cuáles otros recursos para } \\
\text { el control de la temperatura } \\
\text { corporal usa, además de } \\
\text { medicamentos para bajar la } \\
\text { fiebre en el niño? }\end{array}$} & Paños tibios & 89 & 44,5 \\
\hline & Baño de agua & 74 & 37 \\
\hline & fría/tibia/esponja fría & & \\
\hline & Solo utiliza medicamentos & 30 & 15 \\
\hline & Otro: aromática, curar de & & \\
\hline & espanto, nada & 7 & 3,5 \\
\hline \multirow{5}{*}{$\begin{array}{l}\text { ¿Qué instrumento utiliza para } \\
\text { determinar la dosis correcta } \\
\text { del medicamento para bajar } \\
\text { la fiebre? }\end{array}$} & Cuchara de medición & 106 & 53 \\
\hline & especifica o la jeringa & & \\
\hline & específica del medicamento & & \\
\hline & $\begin{array}{l}\text { Cuchara o jeringa de } \\
\text { medición de otros fármacos }\end{array}$ & 78 & 39 \\
\hline & Cucharadas o cucharitas & 16 & 8 \\
\hline \multirow[t]{4}{*}{$\begin{array}{l}\text { Cuando usted va a la farmacia } \\
\text { a conseguir el antibiótico } \\
\text { para su hijo, usted usualmente: }\end{array}$} & $\begin{array}{l}\text { Tiene una fórmula de } \\
\text { antibiótico de su pediatra, } \\
\text { Sólo administra el que } \\
\text { manda el médico }\end{array}$ & 167 & 83,5 \\
\hline & $\begin{array}{l}\text { Decide por su cuenta que } \\
\text { su hijo necesita un } \\
\text { antibiótico por su } \\
\text { experiencia previa }\end{array}$ & 20 & 10 \\
\hline & $\begin{array}{l}\text { Decide usarlo de acuerdo } \\
\text { a la experiencia de otro }\end{array}$ & 10 & 5 \\
\hline & Ninguna & 3 & 1,5 \\
\hline En general, ¿ Usted le da & $\mathrm{SI}$ & 25 & 12,5 \\
\hline $\begin{array}{l}\text { antibióticos a su hijo enfermo } \\
\text { sin consultar a su médico? }\end{array}$ & $\mathrm{NO}$ & 175 & 87,5 \\
\hline $\begin{array}{l}\text { En general, ¿ Usted insiste en } \\
\text { que le prescriban algún }\end{array}$ & $\mathrm{SI}$ & 18 & 9 \\
\hline $\begin{array}{l}\text { antibiótico a su hijo, a pesar de } \\
\text { que el médico no lo considere } \\
\text { necesario por el momento? }\end{array}$ & $\mathrm{NO}$ & 182 & 91 \\
\hline En general, ¿Usted usa & SI & 31 & 15,5 \\
\hline $\begin{array}{l}\text { antibiótico basado en la } \\
\text { consulta al farmaceuta? }\end{array}$ & $\mathrm{NO}$ & 169 & 84,5 \\
\hline
\end{tabular}

En las creencias de los padres de cómo tratar la fiebre de sus hijos (Tabla 4), el 78\% (157) de los padres refiere que no todos los niños con fiebre requieren antibiótico. Dentro de las terapias tradicionales para tratar la fiebre el 69\% (138) respondió que no utiliza nada tradicional, pero el $31 \%$ (62) continúa utilizando algún tipo de terapia como bañar con agua de hierbas, dar a tomar mezclas, soplar alcohol en piel entre otras. El 73.5\% (147) de los padres cree que unos de los efectos secundarios de la fiebre son las convulsiones y el 8,5\% (17) refiere que puede causar la muerte.

Tabla 4. Creencias de los padres sobre la fiebre en el servicio de urgencias.

\begin{tabular}{|c|c|c|c|}
\hline Pregunta & Categoría & Frecuencia & $(\%)$ \\
\hline $\begin{array}{l}\text { ¿Piensa usted que los } \\
\text { antibióticos deberían ser }\end{array}$ & $\mathrm{Si}$ & 43 & 21,5 \\
\hline $\begin{array}{l}\text { prescritos a todos los niños } \\
\text { que desarrollen fiebre? }\end{array}$ & No & 157 & 78,5 \\
\hline ¿Utiliza terapias tradicionales & $\mathrm{Si}$ & 62 & 31 \\
\hline para tratar la fiebre de su hijo? & No & 138 & 69 \\
\hline \multirow[t]{6}{*}{$\begin{array}{l}\text { ¿Qué terapias tradicionales usa } \\
\text { para tratar la fiebre de su hijo? }\end{array}$} & $\begin{array}{l}\text { Dar de tomar mezclas } \\
\text { (aromáticas) }\end{array}$ & 12 & 6 \\
\hline & Bañar con agua de hiervas & 29 & 14,5 \\
\hline & Aplicar limón en la piel & 9 & 4,5 \\
\hline & Soplar con alcohol la piel & 11 & 5,5 \\
\hline & $\begin{array}{l}\text { Otro (Paños de agua tibia, } \\
\text { no abrigo a mi hijo) }\end{array}$ & 13 & 6,5 \\
\hline & Ninguna & 126 & 63 \\
\hline \multirow{4}{*}{$\begin{array}{l}\text { ¿Qué efectos secundarios } \\
\text { puede causar la fiebre si } \\
\text { usted no la trata? }\end{array}$} & Convulsión & 147 & 73,5 \\
\hline & Daño cerebral & 17 & 8,5 \\
\hline & Muerte & 17 & 8,5 \\
\hline & $\begin{array}{l}\text { Otro (no sucede nada, } \\
\text { deshidratación) }\end{array}$ & 19 & 9,5 \\
\hline
\end{tabular}

En la Tabla 5, se encuentra la relación entre variables sociodemográficas y conocimientos, manejos y creencias de la fiebre, en el servicio de urgencias.

Acerca de los conocimientos de los padres, se obtuvo relación significativa ( $\mathrm{p}$-valor prueba $x^{2}<0,05$ ) con las siguientes variables: Se identificó que los padres con edades entre 25 - 33 midieron más la temperatura en axila. Los padres que tiene educación secundaria y universitaria toman la temperatura en la axila. La mayoría de padres con estudios universitarios conocen el concepto de fiebre (temperatura $>38^{\circ} \mathrm{C}$ ) comparados con los de menor nivel educativo. Y consultan más frecuentemente con esta temperatura al médico, mientras los de menor nivel educativo desconocen aque temperatura deben consultar. 
Tabla 5.Variables sociodemográficas de los padres, que se relacionan con los conocimientos, manejos y creencias de la fiebre, en el servicio de urgencias.

\begin{tabular}{|c|c|c|}
\hline CONOCIMIENTOS & Variable a & Schi2 \\
\hline Pregunta & Relacionar & (Valor deP) \\
\hline $\begin{array}{l}\text { Cuando usted toma una temperatura de } \\
\text { un niño menor de seis años, } \\
\text { ¿cuál es el mejor lugar? }\end{array}$ & Edad de los padres & $0,03 *$ \\
\hline -Axila - boca - recto - no sé. & Nivel educativo & $0,00 *$ \\
\hline $\begin{array}{l}\text { ¿Por encima de que temperatura } \\
\text { considera usted que su niño tenga fiebre? } \\
\text { - mayor de } 38 \mathrm{C}^{\circ},-36-27.9,- \text { no sé. }\end{array}$ & Nivel educativo & $0,00^{*}$ \\
\hline $\begin{array}{l}\text { Si su hijo tiene fiebre alta. ¿Hasta cuanto } \\
\text { espera antes de llamar o ir al médico? } \\
\text { - mayor de } 38 C^{\circ},-36-27.9, \text { - no sé. }\end{array}$ & Nivel educativo & $0,003^{*}$ \\
\hline $\begin{array}{l}\text { ¿Cómo se calcula la dosis correcta de } \\
\text { medicamentos para bajar la fiebre a dar } \\
\text { a su hijo? }\end{array}$ & Nivel educativo & $0,01^{*}$ \\
\hline $\begin{array}{l}\text { De acuerdo con el medicamento } \\
\text { que mi pediatra me había aconsejado } \\
\text { previamente, Decido por mí mismo lo } \\
\text { que yo creo que es correcto, Ilamo a mi } \\
\text { pediatra y pregunto, Consulta al } \\
\text { farmacéutico, Consulta a otras personas. }\end{array}$ & $\begin{array}{l}\text { Estrato } \\
\text { socioeconómico }\end{array}$ & $0,00^{*}$ \\
\hline $\begin{array}{l}\text { Usted le administra antibiótico a su hijo: } \\
\text { Cuando el médico le dice que le administra, } \\
\text { cuando tiene fiebre, cuando usted sospecha } \\
\text { una infección. }\end{array}$ & Edad de los padres & $\begin{array}{l}0,01^{*} \\
0,03^{*}\end{array}$ \\
\hline \multicolumn{3}{|l|}{ CREENCIA } \\
\hline $\begin{array}{l}\text { ¿Piensa usted que los antibióticos deberían } \\
\text { ser prescritos a todos los niños que } \\
\text { desarrollen fiebre? } \\
\text { si - no }\end{array}$ & Nivel educativo & $0,00^{*}$ \\
\hline $\begin{array}{l}\text { ¿Utiliza terapias tradicionales para tratar la } \\
\text { fiebre de su hijo? } \\
\text { Si - no }\end{array}$ & Número de hijos & $0,01^{*}$ \\
\hline $\begin{array}{l}\text { ¿Utiliza terapias tradicionales para tratar } \\
\text { la fiebre de su hijo? } \\
\text { si - no }\end{array}$ & Nivel educativo & $0,005^{*}$ \\
\hline MANEJO & & \\
\hline $\begin{array}{l}\text { ¿Qué instrumento utiliza para determinar } \\
\text { la dosis correcta del medicamento para } \\
\text { bajar la fiebre? Cuchara de medición } \\
\text { específico o la jeringa específica del } \\
\text { medicamento, Cuchara o jeringa de medición } \\
\text { de otros fármacos, Cucharadas o cucharaditas. }\end{array}$ & Edad de los padres & $0,001^{*}$ \\
\hline $\begin{array}{l}\text { ¿En general, usted le da antibióticos a su hijo } \\
\text { enfermo sin consultar a su médico? si - no }\end{array}$ & Nivel educativo & $0,01^{*}$ \\
\hline $\begin{array}{l}\text { En general, ¿usted usa antibiótico basado en } \\
\text { la consulta al farmaceuta? } \\
\text { si - no }\end{array}$ & Número de hijos & $0,03^{*}$ \\
\hline
\end{tabular}

En el Manejo de la fiebre, se obtuvo una relación significativa ( $\mathrm{p}$-valor prueba $x^{2}<0,05$ ) con las siguientes variables: A mayor edad de los padres utilizaron más frecuentemente cucharadas o cucharadita para medir la cantidad de medicamentos. La gran mayoría de los padres con estudios universitarios no administran antibiótico sin previa consulta a su médico en comparación con los padres con estudios primarios quienes comparativamente utilizan más antibiótico sin consultar a su médico. Los padres con un hijo medicaron con menor frecuencia antibiótico basados en la consulta al farmaceuta comparados con los que tienen más de 2 hijos.

En las creencias, se obtuvo relación significativa (pvalor prueba $x^{2<0,05)}$ con las siguientes variables: Se encontró que a mayor nivel educativo hay una relación significativa, con los padres que creen que no debe darse antibiótico para el manejo de fiebre. En cuanto a las terapias tradicionales se identificó que los padres con mayor número de hijos, tienden a utilizar más terapias tradicionales comparadas con los que tienen menos hijos. Los padres con estudios universitarios utilizan menos terapias tradicionales para tratar la fiebre en comparación con los padres con estudios primarios.

\section{DISCUSIÓN}

Con respecto a las características socio demográficas, la mayor parte de los padres, eran padres jóvenes, comparado con estudios revisados ${ }^{(6-12)}$. Llama la atención en este estudio, que el $80 \%$ de los padres encuestados, tenían un nivel de educación que no superaba la secundaria, incluso un $21 \%$ del total de los padres, solo reportaron educación básica primaria, a diferencia de lo encontrado en otros estudios donde más de la mitad de los padres referían estudios universitarios ${ }^{(6,8)} \mathrm{O}$ al menos padres con nivel educativo secundario ${ }^{(11,12)}$. Con respecto al estrato socioeconómico, la mayoría de los padres encuestados se encuentran entre estrato 1 y 2 , dado que esta la población que más consulta en esta institución. Contrastado con la literatura que muestra un nivel de ingresos medio ${ }^{(8)}$, otros estudios no tuvieron en cuenta el nivel de ingresos de los padres $^{(6-12)}$.

En cuanto a los conocimientos de los padres, encontramos que muchos padres consideran que la fiebre es una enfermedad en símisma y por tanto su objetivo es curar a los niños de la fiebre y no de la enfermedad que la produce $(9,13)$.

Se encontró en este estudio que la mayoría de padres 
con estudios universitarios conocen el concepto de fiebre, comparados con los de menor nivel educativo $(\mathrm{p}=0,00), \quad \mathrm{y}$ además que los padres con estudios universitarios consultan más frecuentemente con esta temperatura al médico, mientras los de menor nivel educativo desconocen a que temperatura deben consultar; nuestro estudio no es el primero en encontrar estas relaciones entre nivel educativo y conocimiento de la definición de la fiebre, estudios como el de Poirier et al ${ }^{(13)}$, ya lo han reportado previamente.

Para la toma de temperatura se encontró que los padres que tiene educación secundaria y universitaria prefieren tomar la temperatura en la axila $(p=0,00)$ comparados con los que tienen estudios primarios, siendo este el lugar el recomendado para la toma de temperatura en casa.

Este estudio mostró que a mayor nivel educativo y mayor estrato socioeconómico los padres realizan la elección de la dosis del medicamento basados en el consejo previo de un médico. Aunque no se encontraron en otros estudios la relación de estas variables, se menciona que la mayoría de padres realizan la administración de medicamentos basados en la prescripción médica ${ }^{(8)}$. Encontramos que los padres con su primer hijo administran antibiótico guiados por una recomendaciones médica con mayor frecuencia que los padres con 2 hijos o más, no encontramos resultados similares, pero se plantea la hipótesis de que los padres con un solo hijo buscan más el apoyo de un galeno para administrar medicamentos a sus hijos por falta de experiencia previa.

En relación a los hallazgos de nuestro estudio, encontramos similitud con lo revisado en la literatura, con una relación entre el nivel educativo, el estado socioeconómico y los conocimientos y actitudes de los padres frente a la fiebre, que tienden a mostrar una elección más acertada con respecto al manejo de la fiebre en los niños.

Dentro del tratamiento no farmacológico de la fiebre, se encuentra que el baño o esponja con agua tibia son medios físicos momentáneos, proporcionan una leve disminución de la temperatura corporal, y no parece ofrecer ventajas comparadas con los medicamentos antipiréticos ${ }^{(14)}$; en este estudio la mayoría de los padres, utilizan dichos medios físicos; estos hallazgos son consistentes con otros estudios revisados ${ }^{(15-19)}$; a pesar de la poca evidencia de su eficacia, consideramos que su uso frecuente muestra indirectamente el grado de ansiedad y angustia que produce la presencia de fiebre en sus hijos y por lo tanto buscan medidas alternas para bajar la temperatura.

En el presente estudio el $87 \%$ no administran antibiótico a su hijo enfermo sin que un médico lo prescriba, sin embargo llama la atención que el $12,5 \%$ si lo hacen siendo un factor de riesgo para resistencia bacteriana ${ }^{(20,21)}$, no se encontró relación estadística entre estas variables y número de hijos o factores sociodemográficos entre la bibliografía revisada.

La utilización de un dosificador especifico permite evitar errores en la administración de la dosis del medicamento(7); un poco más de la mitad de los padres encuestados en este estudio utilizan la jeringa o cuchara dosificadora del medicamento, resultados similares fueron encontrados en otros estudios ${ }^{(16,17,22)}$.Es importante mencionar que en este estudio, a mayor nivel educativo hay un porcentaje más alto de padres que creen que no debe darse antibiótico para el manejo de fiebre ( $p$-valor prueba $\left.x^{2}<0,05\right)$. En la literatura revisada no se encontraron estudios que relacione estas variables, sin embargo en un estudio realizado por Kabakus et al, el 21,48\% de las madres administraron antibióticos para tratar la fiebre sin consultar a medico .

En cuanto a las terapias tradicionales se encontró que los padres con mayor número de hijos tienden a utilizar más este tipo de terapias como: Dar de tomar mezclas (aromáticas), bañar con agua de hierbas, aplicar limón en la piel, soplar con alcohol la piel. Comparados con los tienen menos hijos (p-valor prueba $\left.x^{2}<0,05\right)$. Teniendo en cuenta que es parte fundamental de cualquier comunidad el respeto hacia las tradiciones culturas, es importante que el equipo de salud no las desconozca ni las rechace, sino que las comprenda para entablar una comunicación efectiva de tal forma que las madres y cuidadores no se sientan agredidos y se les permita la continuidad de su legado cultural. Como lo 
reafirma Noguera, quien refiere que si el personal de salud se da cuenta de la connotación y significado del valor del legado cultural, respetará las creencias y prácticas para ofrecer desde el equipo de salud un cuidado congruente partiendo del saber profesional y así mismo se disminuirán los conflictos entre el profesional de la salud y su familia. En algunos estudios revisados se encontró que todavía el conocimiento de la fiebre y las enfermedades está muy asociado con culturas, tradiciones y costumbres de las comunidades. Es importante mencionar que los padres encuestados con niveles educativos superiores utilizan menos terapias tradicionales para tratar la fiebre en comparación con los padres con estudios primarios ( $\mathrm{p}$-valor prueba $\left.x^{2}<0,05\right)$. Este es un resultado novedoso, debido a que en la literatura revisada para esta investigación no se encontró relación de esta variable con otras.

Se debe tener en cuenta que existen algunas limitaciones. Este tipo de estudio únicamente permite evaluar los pacientes que llegaron al hospital, no se tuvieron en cuenta otros centros hospitalarios. La mayor población que consultó a esta institución fueron estratos socioeconómicos bajos (estrato 1 y 2).

\section{CONCLUSIONES}

Se encontró que la población encuestada en su mayoría fueron padres jóvenes, un gran porcentaje desempleados y de estrato socioeconómico 1 y 2 , con un nivel educativo de secundaria y en su mayoría procedentes de zona urbana.

En cuanto a los conocimientos con respecto a la fiebre aún se encuentran vacíos en los conceptos de fiebre y la toma de temperatura; con respecto a la decisión de administrar medicamentos, los padres se basan en consultas previas al médico.

En cuanto al manejo de la fiebre, un gran porcentaje de los padres utilizan medios físicos concomitante con el uso de antipiréticos, llama la atención que aún persiste la automedicación de antibióticos, aunque este hallazgo se presentó con baja frecuencia.

Con respecto a las creencias, la mayoría de los padres no utilizan terapias tradicionales para tratar la fiebre, sin embargo aún en nuestra región siguen estando arraigadas y presentes las terapias tradicionales para el manejo de fiebre, una tercera parte de los encuestados consideran que la fiebre produce convulsiones e incluso un pequeño porcentaje que puede desencadenar la muerte.

En cuanto a la exploración de la relación de las variables estudiadas hallamos que las variables sociodemográficas que tuvieron mayor relación estadísticamente significativa fueron: nivel educativo, estrato socioeconómico, número de hijos y edad de los padres, que influyeron en el conocimiento, manejo y creencias de los padres hacia la fiebre de sus hijos.

4. Kliegmassn RM, Stanton B, Geme JS, Schor NF, Behrman RE. Nelson textbook of pediatrics. New York: Elsevier Health Sciences; 2015.

5. Sá ACMG-N de, Silva RM, Capanema FD, Gonçalves LAO, Rocha RL. Febre Infantil e seu Manejo pelos Pais: Análise Quantitativa. Rev bras ciênc saúde. 2018; 22(2):117-24.

6. Kelly M, Sahm LJ, Shiely F, O'Sullivan R, De Bont EG, Mc Gillicuddy A, et al. Parental knowledge, attitudes and beliefs on fever: A cross-sectional study in Ireland. BMJ 
Open. 2017;7(7):1-7.

7. Chiappini E, Principi N, Longhi R, Tovo PA, Becherucci $\mathrm{P}$, Bonsignori $\mathrm{F}$, et al. Management of fever in children: Summary of the Italian pediatric society guidelines. Clin Ther [Internet]. 2009 [Citado el 6 de Abr de 2018]; 31(8):1826-43. doi: http://dx.doi.org/10.1016/j.clinthera. 2009.08.006

8. Zyoud SH, Al-Jabi SW, Sweileh WM, Nabulsi MM, Tubaila MF, Awang R, et al. Beliefs and practices regarding childhood fever among parents: A crosssectional study from Palestine. BMC Pediatr. 2013; 13:66.

9. Kohl KS, Marcy SM, Blum M, Jones MC, Dagan R, Hansen J, et al. Fever after Immunization: Current Concepts and Improved Future Scientific Understanding. Clin Infect Dis [Internet]. 2004 [Citado el 5 de Mar de 2018]; 39(3):389-94. doi: http://cid.oxfordjournals.org/lookup/do i/10.1086/422454

10. Tessler H, Gorodischer R, Press J, Bilenko N. Unrealistic concerns about fever in children: The influence of cultural-ethnic and sociodemographic factors. Isr Med Assoc J. 2008; 10(5):346-9.

11. Sahm LJ, Kelly M, Mccarthy S, Sullivan RO, Shiely F, Janne R. Knowledge, attitudes and beliefs of parents regarding fever in children: a Danish interview study. 2016; 105(1):69-73.

12. Arica SG, Arica V, Onur H, Gülbayzar S, Da H, Obut Ö. Knowledge, attitude and response of mothers about fever in their children. Emerg Med J. 2012;29(12):1-6.

13. Poirier MP, Collins EP, McGuire E. Fever phobia: A survey of caregivers of children seen in a pediatric emergency department. Clin Pediatr (Phila). 2010; 49(6):530-4.

14. Sanjuanelo AB. Fiebre : actualización en el uso de antipiréticos. Precop. 2013; 11(4):26-35.

15. Crocetti M, Moghbeli N, Serwint J. Fever Phobia Revisited: Have Parental Misconceptions About Fever Changed in 20 Years?. Pediatrics [Internet]. 2001 [Citado el 13 de Mar de 2018];107(6):1241-6. doi: http://pediatrics. aappublications.org/cgi/doi/10.1542/peds.107.6.1241

16. Athamneh L, El-Mughrabi M, Athamneh M, Essien EJ, Abughosh S. Parents' Knowledge, Attitudes and Beliefs of Childhood Fever Management in Jordan: a CrossSectional Study. J Appl Res Child [Internet]. 2014 [Citado el 10 de Abr de 2018]; 5(1):1-31. Recuperado a partir de: http://digitalcommons.library.tmc.edu/childrenatrisk/vol 5/iss1/8/?utm_source $=$ digitalcommons.library.tmc.edu $\%$ 2Fchildrenatrisk\%2Fvol5\%2Fiss1\%2F8\&utm_medium=P DF\&utm_campaign=PDFCoverPages

17. Al-Abdel Jalil HK, Jumah NA A-BA. Mothers' knowledge, fears and self-management of fever: a crosssectional study from the capital governorate in Kuwait. Kuwait Med J. 2007;39(4):349-54.

18. Monterrosa-Castro Á, Arias-Martínez M, Armando Echandía C. Detección, manejo y percepción materna de la fiebre en niños Cali - Colombia. Rev Colomb. [Internet]. 2012 [Citado el 15 de Mar de 2018]; 60(1):40-9. Recuperado apartir de: http://www.scielo.org.co/pdf/rcog/v63n1/v63 n1a03.pdf

19. Harbarth S, Balkhy HH, Goossens H, Jarlier V, Kluytmans J, Laxminarayan R, et al. Antimicrobial resistance: One world, one fight! Antimicrob Resist Infect Control [Internet]. Antimicrobial Resistance and Infection Control. 2015 [Citado el 16 de Mar de 2018]; 4(1):1-15. doi: http://dx.doi.org/10.1186/s13756-015-0091-2

20. Al-Abdel Jalil HK, Jumah NA A-BA. Mothers' knowledge, fears and self-management of fever: a crosssectional study from the capital governorate in Kuwait. Kuwait Med J.2007;39(4):349-54.

21. Harbarth S, Balkhy HH, Goossens H, Jarlier V, Kluytmans J, Laxminarayan R, et al. Antimicrobial resistance: One world, one fight! Antimicrob Resist Infect Control [Internet]. Antimicrobial Resistance and Infection Control. 2015 [Citado el 18 de Abr de 2018]; 4(1):1-15. doi: http://dx.doi.org/10.1186/s13756-015-0091-2

22. WHO. Antimicrobial resistance. Global report on surveillance [Internet]. World Health Organization; 2014 [Citado el 3 de May de 2018]. Recuperado apartir de: https://www.who.int/drugresistance/documents/surv eill ancereport/en/

23. Pérez-Conesa MC, Pina IS, Manonellas SR, Esparza AT, García V, López M. Análisis de los cuidados y los conocimientos parentales sobre la fiebre en la infancia. Atención Primaria [Internet]. 2017 [Citado el 24 de Mar de 2018]; 49(8):4-11. doi: http://pediatrics.aappublications. org/cgi/doi/10.1542/peds.107.6.1241

24. Noguera NY, Rodriguez MR. Aprendiendo a cuidar al recién nacido: un cuidado congruente con la cultura. Av en Enfermería [Internet]. 2010 [Citado el 24 de Abr de 2018];26(1):103-11. Recuperado apartir de: http://revistas.unal.edu.co/index.php/avenferm/article/vi ew/12890/13648 